\title{
Adaptive Feature and Score Level Fusion Strategy using Genetic Algorithms
}

\author{
Wael Ben Soltana, Mohsen Ardabilian, Liming Chen and Chokri Ben Amar \\ MI Department, LIRIS Laboratory, CNRS 5205, Ecole Centrale de Lyon, 69134, Lyon, France \\ \{wael.ben-soltana, Mohsen.Ardabilian,Liming.Chen\}@ec-lyon.fr, chokri.benamar@ieee.org
}

\begin{abstract}
Classifier fusion is considered as one of the best strategies for improving performances upon general purpose classification systems. On the other hand, fusion strategy space strongly depends on classifiers, features and data spaces. As the cardinality of this space is exponential, one needs to resort to a heuristic to find out a sub-optimal fusion strategy. In this work, we present a new adaptive feature and score level fusion strategy (AFSFS) based on adaptive genetic algorithm. AFSFS tunes itself between feature and matching score levels, and improves the final performance over the original on two levels, and as a fusion method, not only it contains fusion strategy to combine the most relevant features so as to achieve adequate and optimized results, but also has the extensive ability to select the most discriminative features. Experiments are provided on the FRGC database and show that the proposed method produces significantly better results than the baseline fusion methods.
\end{abstract}

\section{Introduction}

In pattern recognition and computer vision literature, there are many fusion-related research works on different levels. Fusion information is a relatively understudied problem because of practical difficulties. It may cause significant classifier performance losses if the best fusion scheme is not relevantly chosen [4].

Several fusion strategies can be roughly classified into three main categories: fusion at an early stage, fusion at a later stage and hybrid fusion. However, many systems that integrate information at an early stage are believed to be more effective than those that perform integration at a later stage [3]. Therefore, while it is relatively more difficult to achieve in practice, fusion at early stage has drawn more attention in recent years. There are two types of early fusion: fusion at image level (3D image [1] or 3D/2D image [2]), and fusion at feature level [3]. In fact, at the feature level the concatenated feature vectors may contain noisy or redundant data, thus leading to decreased performances of the classifier [4]. In this case, the feature selection procedure is an important step. Several search strategies, which provide one optimal (or near-optimal) subset of features with respect to a certain criterion, have been put forward: exhaustive search $[4,5]$, best individual features (BIF), sequential forward selection (SFS), sequential backward selection (SBS) [4], sequential forward floating search (SFFS) [4,6], sequential backward floating search (SBFS) [4,7] and evolutionary algorithms. Among them, the genetic algorithm (GA) [8] includes a subset of evolutionary algorithms focusing on the application of selection, mutation, and recombination to a population of competing problem solutions. In fusion at later stage, all classifiers are included in the fusion scheme. Since these individual experts may be correlated, it may not be the best scheme to follow. In this case, there are three fusion sub-levels: score match level [9], rank level [10] and decision level [11]. Kittler and al. [15] presented and developed a common theoretical framework for these combining classifiers. At the first level, similarity scores are combined by various techniques [12], for example, Sum Rule, Product Rule, etc. At the second level, sorted lists computed by classifiers are merged based on different approaches such as Borda Count and Logistic Regression [13]. At the third level, all the candidates of the classifiers are fused by adopting several methods [14], i.e., Majority Vote or Majority Vote with maximum confidence. The last category contains intermediate fusion schemes, such as serial fusion and multilevel fusion. The main motivation of the serial or hierarchical architecture [14] is to filter out the most similar $\mathrm{K}$ classes using a simple classifier and then to feed these $\mathrm{K}$ classes into a more complex and powerful second classifier. On the other side, there are 
few works that describe multilevel fusion. In [16], fusion is introduced in both feature level and confidence level for face recognition.

In this work, we propose a general multilevel fusion method that is able to obtain a global sub-optimal solution while lessening the complexity of calculation. The main contributions of this paper are as follows. We propose to use a genetic algorithm with a novel coding strategy for effective feature selection; at the same time an optimal fusion strategy scheme is generated at both feature and matches score levels.

The remainder of this paper is organized as follows: Adaptive feature and score level fusion strategy is introduced in section2, and section 3 presents experimental results. Section 4 concludes the paper.

\section{Adaptive feature and score level fusion strategy (AFSFS)}

The proposed approach (Figure. 1) is based on genetic algorithm, using a novel coding technique, to search the optimal fusion scheme.

\subsection{Algorithm Overview}

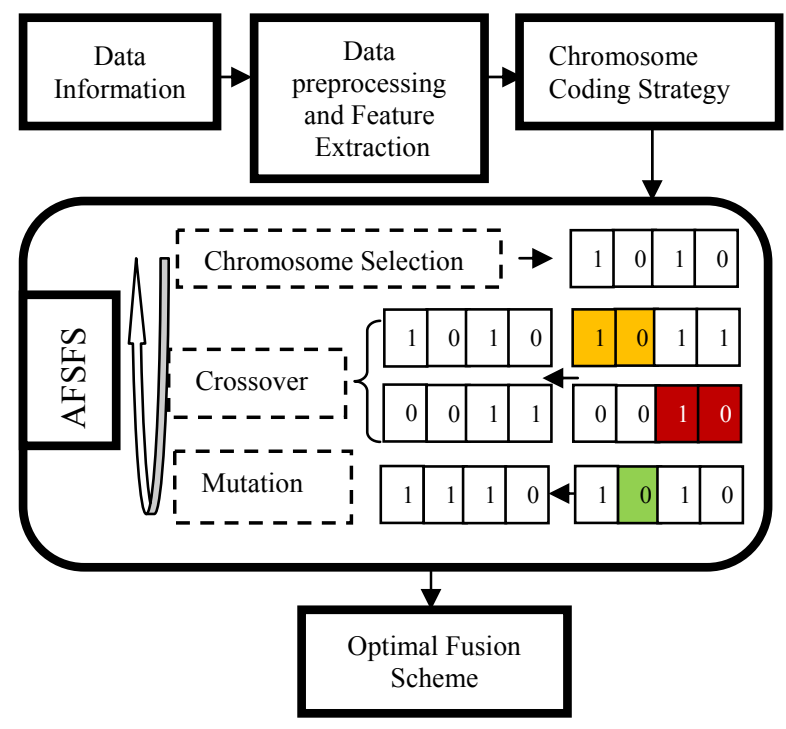

Figure 1. Algorithm Overview

The method consists of two steps. In the first step the data information is preprocessed and features are extracted. For measurement cost and classification accuracy, Linear Discriminant Analysis (LDA) is used to reduce the dimensionality for each feature. The second step finds one subset of features that is optimal with respect to the corresponding fusion scheme. So, all features are coded to form individual "chromosomes" according to the model described in the section 2.2. Furthermore, these chromosomes are used by a genetic algorithm [21] to encode the trial solution for the current problem. Iterative selection, crossover, and mutation were used to make evolve a new population. At each new generation, a new set of chromosomes is produced, using the fittest genes of the previous generation, for a better solution. Assessment of the satisfactory degree of this solution, encoded as individuals, is reflected in the fitness. Also, the individuals with higher fitness have a high probability of being selected and producing offspring. The crossover operator produces better offspring by exchanging the characteristics of the parents. This enables the most efficient characteristics to be concentrated in the same individual. The mutation operator randomly changes the genetic representation of an individual and tends to inhibit the possibility of converging to a local optimum, rather than the global optimum. The evolution is carried out until a desired solution is arrived, or a pre-specified number of iterations are completed. The final solution with higher fitness represents the optimal fusion strategy.

\subsection{Basic Properties of Genetic Algorithm}

We propose a novel coding strategy to select simultaneously the efficient feature and the optimal fusion scheme. This coding strategy consists to divide the chromosome into two parts: Part A and Part B (See Figure. 2). Given $\mathrm{N}$ features, Part A has $N$ gene positions that correspond to each feature, and represented with integer values: 1 implies that the feature is active and used in feature level fusion, 0 implies that the feature is active and used in score level fusion, and -1 implies that the feature is inactive. Part $\mathrm{B}$ codes the fusion model that depends on the number $N_{F}$ of active features at feature level fusion. In this model, we generate all possible combinations. However, we can't create a strategy that contains a single feature and we consider that combinations obtained by permutation are equivalent. Part B is also composed of two parts P1and P2: P1 refers to the model $\mathrm{M}$ and $\mathrm{P} 2$ associates the features in this model.

An example of this representation is illustrated in Figure 2. With a Part A as 1011101-11, we can generate 4 models $\mathrm{M}_{\mathrm{i}}$, with $i$ in $\{1 . .4\}: \mathrm{M}_{1}=(6,0), \mathrm{M}_{2}=$ $(2,4), M_{3}=(3,3), M_{4}=(2,2,2)$. The number of the selected model is represented in the chromosome by its binary code: the model $\mathrm{M}_{2}$ is selected and represented by (010) and two vectors V1, V2 are created by concatenation, $\mathrm{V} 1=[\mathrm{F} 3, \mathrm{~F} 4]$ and $\mathrm{V} 2=[\mathrm{F} 5, \mathrm{~F} 1, \mathrm{~F} 7, \mathrm{~F} 9]$. The fusion strategy corresponds to a score matching level with V1, V2, F2 and F6. The fitness of this strategy is calculated based on performance rate with simple sum rule in score level fusion. Stochastic universal sampling [17] is used to select best 
chromosomes "strategies". Uniform crossover is used only on Part A and random mutation may occur on Part A or Part B of chromosome. Stopping criteria chosen for problem solving is selected from these conditions: 1) either the maximum number of iterations over the terminal number max of generations, 2) the best fitness value beyond the value of fitness limits.

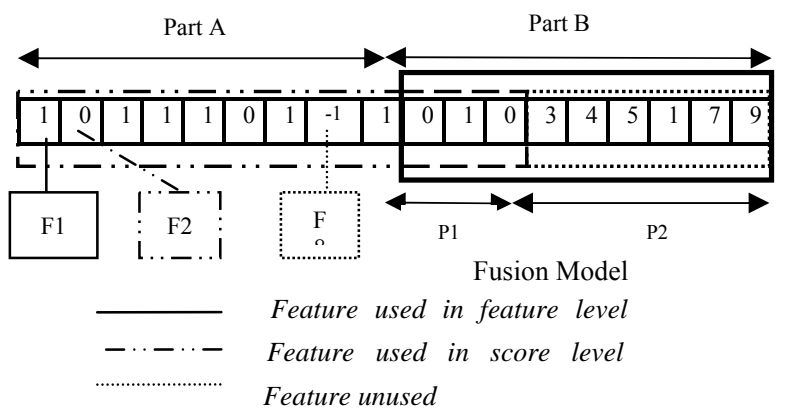

Figure 2. Example of chromosome coding strategy

\section{Experimental Results}

The proposed algorithm is tested in a face recognition application, where the objective is to find an optimal subset of features and their adequate fusion strategy.

\subsection{Database, Experiment Settings and Feature Extracted}

The FRGC [18] database was chosen for our experiments. Each face data consists of one 3D face model and its registered 2D color image. 3D faces are preprocessed with techniques in [22]. FRGC v1.0 dataset is used for estimating LDA parameters while FRGC v2.0 is used for test. In the first step, 116 subjects having each 4 face models were selected from FRGC v1.0 to train subspace based approaches such as estimating LDA parameters. One 3D face scan with a neutral expression was selected from each subject to make a gallery of 50 subjects and 472 (3D) face scans were treated as probes. Furthermore, we have used 3D features. In fact, we have considered the raw pointcloud representation of 3D face model as well as geometric features containing normal (Nor Vec), binormal (BiN Vec), tangent vector (Tang Vec) [19] and curvature related features that have the potential for a higher accuracy to describe the surface based events. Four categories of curvature-based features are extracted. The first two types rely on main directions corresponding to maximum (Max Curv) and minimum (Min Curv) curvatures [20]. The last two are their derivatives, i.e., the mean (Mean Curv) and Gaussian (Gauss Curv) curvatures. We further investigated another type of 3D feature based on the anthropometric (Anthr Mes) approach which advocates extracting a signature from some anthropometric points considered the most relevant. So, Part A of chromosome is organized as follows: \{Tang Vec, BiN Vec, Nor Vec, Gauss Curv, Max Curv, Mean Curv, Min Curv, Anthr Mes\}.

\subsection{Results and Analysis}

LDA is applied to reduce dimensionality of all features. One similarity measure of each feature was computed with Nearest Neighbor (NN). Table 1 displays the performance of each feature. As in the table, the best rank-one recognition rate is provided by tangent vectors with $86.22 \%$.

Table 1. Rank-one recognition rate of individual type of feature on the FRGC v2.0 database

\begin{tabular}{|l|c|l|l|l|c|}
\hline & NN & & \multicolumn{1}{|c|}{ NN } & & NN \\
\hline Max Curv & 73.98 & Mean Curv & 79.59 & BiN Vec & 79.59 \\
\hline Gauss Curv & 62.76 & Anthr Mes & 64.8 & Nor Vec & 79.59 \\
\hline Min Curv & 73.98 & Tang Vec & 86.22 & & \\
\hline
\end{tabular}

Table 2. Rank-one recognition rates with different fusion schemes

\begin{tabular}{|c|c|}
\hline & $\begin{array}{l}\text { Recognition } \\
\text { Rate }\end{array}$ \\
\hline SBFS-based classifier selection [6] & $91.84 \%$ \\
\hline 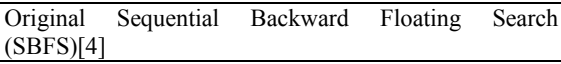 & $90.31 \%$ \\
\hline $\begin{array}{l}\text { Adaptive feature and score level fusion strategy } \\
\text { (AFSFS) }\end{array}$ & $92.35 \%$ \\
\hline
\end{tabular}

With a gain of 5.62 percent, the improvement is achieved by SBFS-based classifier selection [6] (See Table2). In fact, the near optimal subset found by SBFS-based classifier selection for simple sum technique is \{Tang Vec, BiN Vec, Mean Curv, Anthr Mes \}. The same subset is produced by AFSFS from the relevant chromosomes in the following form: Part A: 00-1-1-10-10, Part B: P1: 000001. In our work, the optimal strategy fusion is generated after 50 generations, each generation containing 50 chromosomes, and coded as follows. Part A: 00111010, Part B: [P1: 000010, P2:7, 4, 3, 5]. The selected strategy consists firstly to concatenate \{Nor Vec, Gauss Curv $\}$ in vector $\mathrm{V}_{1}$, \{Max Curv, Min Curv $\}$ in vector $V_{2}$. Secondly, we use this optimal subset $\left\{\mathrm{V}_{1}, \mathrm{~V}_{2}\right.$, Tang Vec, BiN Vec, Mean Curv, Anthr Mes $\}$ in score level fusion to produce the best rank-one recognition rate $92.35 \%$. As we can see in table2, Original Sequential Backward Floating Search [4] represents a feature-selection method which achieves a $90.31 \%$ rank-one recognition rate. The proposed genetic algorithm improves rank-one recognition accuracy as compared with other methods $[4,6]$ in two steps: selecting the most discriminative features and proposing an optimized fusion strategy. 


\section{Conclusions and Future Works}

In this paper we propose a framework of fusion strategies that is able to go far beyond the classical early and late fusion. A genetic algorithm is used to find a sub-optimal fusion scheme. This technique is a powerful global optimization method which is based on natural selection and genetics mechanisms. However, it is important to use the suitable coding strategy. Therefore, a new coding technique is developed and shows its effectiveness through experiments on FRGC v2.0 dataset. In fact, the proposed method AFSFS tunes itself between feature and matching score levels, and produces significantly better results than Original Sequential Backward Floating Search (SBFS) and SBFS-based classifier selection.

In future works, we intend to extend this fusion scheme in order to generate the best model featureclassifier-fusion (FCF). This can be possible if fitness function is dynamic and can select the best classifier for each fusion method. We plan as well to apply this framework to other applications in pattern recognition.

\section{References}

[1] Theodoros Papatheodorou, Daniel Rueckert. Evaluation of Automatic 4D Face Recognition Using Surface and Texture Registration. Automatic Face and Gesture Recognition, 2004, 17-19 May 2004 Page(s): 321 - 326.

[2] Gede Putra Kusuma and Chin-Seng Chua. Image Level Fusion Method for Multimodal 2D $+3 \mathrm{D}$ Face Recognition. ICIAR 2008: 984-992

[3] A. S. Mian, M. Bennamoun, and R. Owens. Keypoint detection and local feature matching for textured 3D face recognition. International Journal of Computer Vision, 2008.

[4] A. K. Jain, R. P. W. Duin, J. Mao: Statistical Pattern Recognition: A Review. IEEE Trans. Pattern Anal. Mach. Intell. 22(1): 4-37 (2000)

[5] P.M. Narendra and K. Fukunaga, A Branch and Bound Algorithm for Feature Subset Selection. IEEE Transactions on Computers, , vol. C-26, issue 9, pp. 917922, Sept 1977.

[6] B. Gökberk, H. Dutağacı, A. Ulaş, L. Akarun, and B. Sankur. Representation plurality and fusion for 3D face recognition. IEEE Trans. on Systems Man and Cybernetics-Part B: Cybernetics, 38(1):155-173, 2008.

[7] D. Ververidis, C. Kotropoulos. Fast and accurate sequential floating forward feature selection with the Bayes classifier applied to speech emotion recognition. Signal Processing 88(12): 2956-2970 (2008).

[8] D. Ruta, and B. Gabrys, Classifier Selection for Majority Voting. Special issue of the journal of INFORMATION FUSION on Diversity in Multiple Classifier Systems, vol. 6, issue 1, pp. 63-81, 1 March 2005.
[9] J. Cook, M. Cox, V. Chandran, and S. Sridharan. Robust 3D face recognition from expression categorization. International Conference on Biometrics, 271-280, 2007.

[10] B. Gökberk, A. Salah, and L. Akarun. Rank-based decision fusion for 3D shape-based face recognition. International Conference on Audio- and Video-Based Biometric Person Authentication, 1019-1028, 2005.

[11] T. Faltemier, K. Bowyer, P. Flynn. 3D face recognition with region committee voting. International Symposium on 3D Data Processing, Visualization, and Transmission, 2006.

[12] A. Godil, S. Ressler, and P. Grother. Face recognition using 3D facial shape and color map information: comparison and combination. Biometric Technology for Human Identification, SPIE, 5404:351-361, 2005.

[13] Md. M. Monwar, M. Gavrilova. FES: a system for combining face, ear, and signature biometrics using rank level fusion. International Conference on Information Technology: New Generations, 922-927, 2008.

[14] B. Gökberk and L. Akarun. Comparative analysis of decision level fusion algorithms for 3D face recognition. International Conference on Pattern Recognition, 2006.

[15] J. Kittler, M. Hatef, R. P. W. Duin, J. Matas. On Combining Classifiers. IEEE Trans. Pattern Anal. Mach. Intell. 20(3): 226-239 (1998).

[16] Congcong Li, Guangda Su, Yan Shang, Yingchun Li, and Yan Xiang. Face Recognition Based on Pose-Variant Image Synthesis and Multi-level Multi-feature Fusion. AMFG 2007: 261-275

[17] J. E. Baker. Reducing Bias and Inefficiency in the Selection Algorithm. Proceedings of the Second International Conference on Genetic Algorithms and their Application, 14-21,1987.

[18] P. J. Phillips et al. Overview of the face recognition grand challenge. IEEE International Conference on Computer Vision and Pattern Recognition, I:947-954, 2005.

[19] S.W. Zucker. Differential geometry from the frenet point of view: boundary detection, stereo, texture and color. Handbook of Mathematical Models in Computer Vision, N. Paragios, Y. Chen, and O. Faugeras, eds., Springer, 2005

[20] H. Tanaka, M. Ikeda, and H. Chiaki. Curvature-based face surface recognition using spherical correlation. IEEE International Conference on Automatic Face and Gesture Recognition, 372-377, 1998.

[21] M. Sedaaghi, D. Ververidis and C. Kotropoulos. Improving speech emotion recognition using adaptive genetic algorithms," in Proc. European Signal Processing Conference (EUSIPCO),Polland, 2007.

[22] P. Szeptycki, M. Ardabilian, and L. Chen. A coarse-tofine curvature analysis-based rotation invariant $3 \mathrm{D}$ face landmarking. International Conference on Biometrics: Theory, Applications and Systems, 2009. 